\title{
A specialised cardiorespiratory team approach in the intensive care management of COVID-19 patients: benefit on mortality, diagnosis and management
}

\author{
Authors: Asad Anwar, ${ }^{\mathrm{A}}$ Nordita Ramos-Bascon, ${ }^{\mathrm{B}}$ A Agatha Crerar-Gilbert, ${ }^{\mathrm{C}}$ Natalie Barnes ${ }^{\mathrm{D}}$ and Brendan Madden ${ }^{\mathrm{E}}$
}

\section{Background}

During the coronavirus pandemic, our intensive care units were faced with large numbers of patients with an unfamiliar disease. To support our colleagues and to assist with diagnosis and treatment, we developed a specialist team.

Methods

The acute respiratory disease support team reviewed 44 consecutive patients referred from the intensive care and coordinated therapies for pulmonary hypertension, pulmonary thrombosis, evolving lung fibrosis and large airway intervention.

\section{Results}

The mortality for this group was significantly lower (34\%) than the total group admitted to critical care as a whole (51\%) and for those not reviewed by the team (55\%; $p=0.012$ ). Pulmonary hypertension was present in $84 \%$ of the patients and pulmonary thrombosis in $52 \%$. Thirty-two patients received sildenafil therapy and this was associated with improvement in right heart function in survivors. Ten patients with evolving fibrosis and no evidence of sepsis received high-dose steroid therapy with excellent effect. Five patients developed airway complications requiring intervention. Short time on mechanical ventilation was associated with a poorer outcome $(p<0.001)$.

\section{Interpretation}

A specialised cardiorespiratory team approach contributes significantly to successful management of severely unwell patients with COVID-19 and offers an important platform for continuity of patient care, education and staff well-being.

KEYWORDS: cardiorespiratory, COVID-19, intensive care, pulmonary hypertension

DOI: $10.7861 /$ clinmed.2020-0788

Authors: A specialist registrar in respiratory medicine, St George's University Hospitals NHS Foundation Trust, London, UK; B pulmonary hypertension clinical nurse specialist, St George's University Hospitals NHS Foundation Trust, London, UK; C Consultant cardiothoracic anaesthetist, St George's University Hospitals NHS

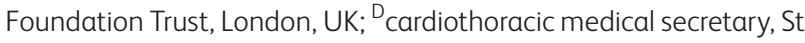
George's University Hospitals NHS Foundation Trust, London, UK; Econsultant cardiothoracic and intensive care physician, St George's University Hospitals NHS Foundation Trust, London, UK

\section{Introduction}

SARS-CoV-2 is responsible for causing COVID-19 and an acute respiratory distress syndrome is a recognised complication. ${ }^{1}$ Acute right heart dysfunction can accompany this and may reflect proximal pulmonary thrombotic elements, peripheral (in situ) thrombosis and myocarditis (Table 1). ${ }^{2-5}$

Recent evidence supports the use of dexamethasone in managing patients with COVID-19. ${ }^{6}$ However, the role for high dose steroid therapy in attempting to reverse or mitigate evolving lung fibrosis is unknown.

Having worked in our 150-bed intensive care unit (ICU) areas from the beginning of the pandemic, we believed that a specialist dedicated team would assist our medical and nursing colleagues, and help identify and treat the various cardiopulmonary pathologies contributing to the critical illness of our patients (Table 1). This new team comprised a professor of cardiothoracic medicine with significant experience in pulmonary hypertension (PH), endo-myocardial biopsy, large airway intervention and cardiothoracic intensive care; a respiratory medicine specialist registrar; a consultant cardiothoracic anaesthetist; a PH nurse specialist; and an experienced cardiothoracic medical secretary. ${ }^{7-9}$ We named this

\begin{tabular}{|c|c|}
\hline $\begin{aligned} \text { > Infection } \\
\text { > viral } \\
\text { > bacterial } \\
\text { > fungal. } \\
\text { > Left heart dysfunction/ } \\
\text { rhythm disturbances. } \\
\text { > Hyper-/hypovolaemia. } \\
>\text { Thrombosis } \\
\text { > in situ } \\
\text { > embolic. } \\
>\text { Immunological response } \\
\text { (humoral and cellular). } \\
\text { > Right heart dysfunction. } \\
\text { > Myocarditis. }\end{aligned}$ & $\begin{array}{l}\text { > Pulmonary hypertension } \\
\text { > hypoxia } \\
\text { > vasoconstriction } \\
\text { > thrombosis } \\
>\text { vascular remodelling } \\
>\text { acute lung injury with loss of } \\
\text { gas transfer area } \\
>\text { pre-existing conditions. } \\
\text { > Pre-morbid cardiorespiratory } \\
\text { diseases and medication. } \\
>\text { Evolving pulmonary fibrosis. } \\
>\text { Current medical comorbidities } \\
\text { eg other organ dysfunction. } \\
>\text { Consequences of mechanical } \\
\text { ventilatory support. }\end{array}$ \\
\hline
\end{tabular}


team the acute respiratory disease support team. The purpose of our team was to provide specialist input for severely unwell patients with COVID-19 early in their disease and to assess for conditions that may respond to specific therapies, including pulmonary vasodilator therapy (PVT) and steroids. We developed a proposal for referral of patients from all the intensive care and high-dependency units within the hospital. There was close communication between our team and colleagues in microbiology and infectious disease.

\section{Methods}

We developed a proposal for referral of patients from all the intensive care and high-dependency units within the hospital and this received universal support from our medical and nursing staff. We were particularly interested in assessing patients with suspected pulmonary thrombosis, patients with pulmonary hypertension, those potentially requiring high-dose steroid therapy and patients who had large airway compromise. All of the patients assessed by our team had been admitted directly to the ICU from the acute medical admission units or had been transferred from other ICUs in the region.

From mid-April 2020 to the end of May 2020, we reviewed all patients referred to us every day. All diagnoses, treatments and interventions were recorded and patients' responses were monitored. A database was created and all clinical information was analysed. Treatment plans were discussed with patients' next of kin and there were further discussions subsequently in the planned outpatient follow-up clinics. Chi-squared test, Fisher's exact test, Wilk normality test, independent samples t-test, Mann-Whitney $U$ test and area under the receiver operating characteristics curve were the statistical methods employed.

We considered 2D echocardiography and computed tomography pulmonary angiography (CTPA) essential diagnostic tests. We were particularly interested in pulmonary thrombosis and, in addition to anticoagulation, prescribed sildenafil as PVT for those patients who had PH (right ventricular dysfunction (RVD) and/ or estimated pulmonary artery systolic pressure (PASP) $>40$ $\mathrm{mmHg}$ ) in association with either proximal thrombotic elements or peripheral (in situ) thrombosis. The dose was titrated with renal function and blood pressure and we considered potential drug interactions.

Steroid therapy was considered for patients whom we believed had an immunologically driven pneumonitis and evolving fibrosis (eg ground glass shadowing, peripheral airspace dilatation and developing traction bronchiectasis) and in whom there was no evidence of intercurrent infection. From experience in the transplant literature and in other immunological conditions, we prescribed intravenous methylprednisolone $1 \mathrm{~g}$ daily on 3 consecutive days followed by oral prednisolone $40 \mathrm{mg}$ daily reducing by $5 \mathrm{mg}$ after every 3 days to zero. $^{10-12}$

Following discharge from the ICU, patients were reviewed each day until hospital discharge. At hospital discharge, a 3-month appointment was given for our new specialist follow-up clinic where patients have a clinical assessment together with an un-encouraged 6-minute walk test, 2D echocardiography, CTPA and full lung function tests. Onward referral was made as appropriate.

\section{Results}

Forty-four critically unwell patients with COVID-19 were referred. There were 34 men (age range 17-77 years; median 59.5 years; interquartile range (IQR) 50.5-64.5) and 10 women (age range 21-80 years; median 59 years; IQR 49-67.5). Thirty-three patients (75\%) had positive PCR swabs for COVID-19, 10 were negative while one patient had indeterminate results (all these 11 patients had clinical, haematological and radiological features suggestive of COVID-19).

Thirty-six patients ( 29 men (median age 59 years; IQR 50-65.5) and seven women (median age 58.5 years; IQR 46-63)) were on mechanical ventilatory support at the time of referral. Twentyfour patients received a tracheostomy and 12 did not. Twentythree patients, 19 men (median age 55 years; IQR 46-63) and four women (median age 62.5 years; IQR 55.25-63.75) survived. Thirteen patients ( 10 men (median age 62.5 years; IQR 59.75-68) and three women (median age 46 years; IQR 21-60)) died. One of the women who died was 21 years old who had significant kyphoscoliosis and bronchiectasis and was declined extracorporeal membrane oxygenation by the local specialist centre. The duration of mechanical ventilatory support for the group as a whole was 3-65 days (median 23.5; IQR 15-43). For those patients who survived the time on mechanical ventilatory support was 3-65 days (median 36; IQR 23-49). For those patients who died the range was 5-29 days (median 18; IQR 8.5-20.5). It should be noted that some of the patients had been on mechanical ventilatory support for 3 weeks before having been reviewed by our team (Fig 1).

In patients who had been on mechanical ventilatory support, there was no significant difference between those patients who survived and those who died with regard to age and gender $(p=0.69)$. However, there was a highly significant difference between the groups with regard to time on ventilatory support with those patients who died having been on a ventilator for a shorter time $(p<0.001)$.

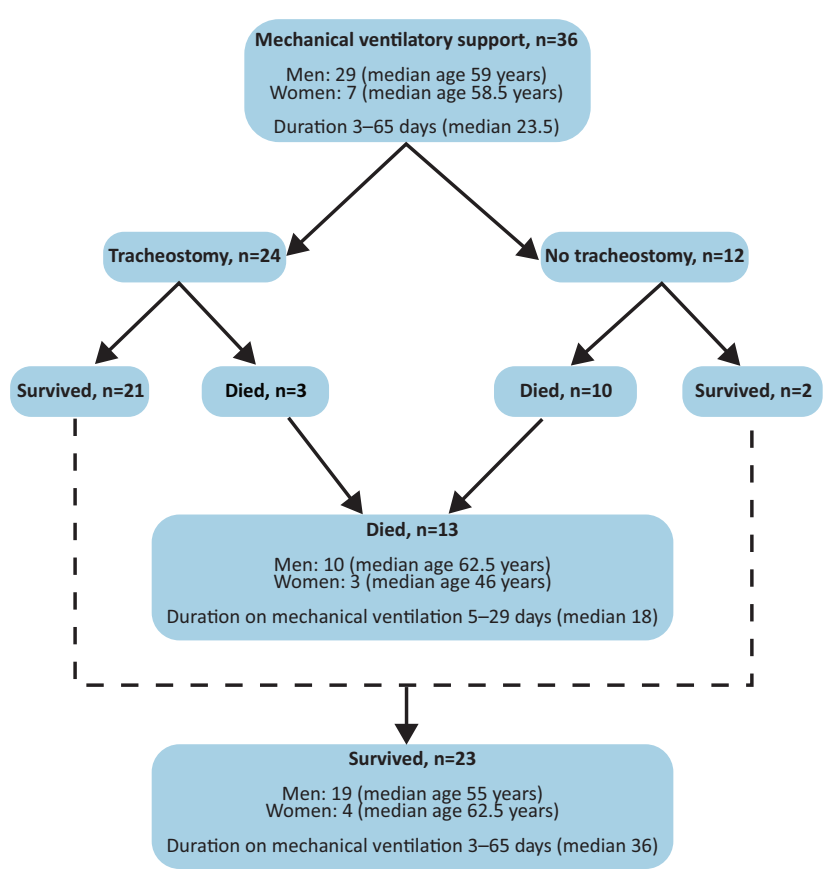

Fig 1. The outcome of patients on mechanical ventilation. 
Thirty-one patients (70\%) were of Black, Asian and minority ethnic (BAME) background and 13 patients were White (30\%). The mortality for the White patients was $2 / 13(15 \%)$ and for those of BAME background was 13/31 (38\%). This difference in mortality was statistically significant ( $p=0.037$ ).

Two-hundred and sixteen patients were admitted with COVID-19 to the ICUs in our hospital between March 2020 and the end of May 2020. One-hundred and ten people (51\%) died. In our cohort, 15 patients died ( $34 \%$ mortality) and the mortality of ICU patients excluding our cohort of patients was $55 \%$. Mortality in the group treated by the specialist team was significantly lower $(p=0.012$ ) when compared with mortality of the ICU patients as a whole and the group of patients in ICU who were not reviewed by our team.

In our group of patients, several pathologies were identified.

\section{Pulmonary thrombosis}

Twenty-three patients ( $52 \%$ ) were diagnosed with pulmonary thrombosis. All received anticoagulation comprising standard treatment dose for pulmonary embolism of low molecular weight or unfractionated heparin depending on renal function with subsequent conversion to a direct oral anticoagulant (DOAC). Twenty-one (91\%) received PVT (sildenafil) because of evidence of raised PASP or RVD on 2D echocardiography. Eight patients had proximal pulmonary thrombosis, including one patient who also had right ventricular clot. Seven patients $(85 \%)$ received thrombolysis for saddle/central pulmonary artery thrombus, heavy clot burden, haemodynamic compromise and/ or signs of RVD. Five of these patients subsequently died: four from multiple organ failure and one from unrelated aspiration pneumonia. Two patients survived and continued sildenafil and anticoagulation. One patient who had proximal pulmonary thrombus without haemodynamic compromise and who had a normal 2D echocardiography assessment did not receive sildenafil and remains well. Fifteen patients had peripheral pulmonary thrombosis. Three patients (20\%) died. In the surviving 12 patients, 11 received sildenafil. All demonstrated a favourable response with regard to $\mathrm{PH}$ and right ventricular function on $2 \mathrm{D}$ echocardiography following the introduction of sildenafil therapy. One further patient with peripheral thrombosis did not receive sildenafil as he did not have evidence of $\mathrm{PH}$ and he is well. No patient experienced any side effects from sildenafil.

\section{RVD and $\mathrm{PH}$}

Thirty-seven (84\%) patients had PH as evidenced by echocardiography showing RVD and/or raised PASP. Sildenafil was used as a first-line agent. We did not use prostacyclin or endothelin receptor antagonists. Five of the 37 patients did not receive sildenafil treatment because three were considered terminal at the time of referral to our team, one had acute pulmonary oedema secondary to myocardial infarction and one was persistently hypotensive. Thirty-two (72\%) patients were therefore identified as appropriate for treatment. Twenty-one of these $(65 \%)$ had pulmonary thrombosis and the remaining 11 had $\mathrm{PH}$ which we believed to be multifactorial (Table 1). Eight of these patients had significant changes on thoracic computed tomography (CT) and we believe that their $\mathrm{PH}$ reflected this. One patient had PH associated with known sarcoidosis and two patients had not had thoracic CT at the time of referral to us because they were considered too unstable to transfer to the radiology department but chest X-ray showed diffuse bilateral infiltrates in keeping with severe SARS-CoV-2 infection. Sildenafil was commenced at a dose of $25 \mathrm{mg}$ three times a day (TDS) increasing to $50 \mathrm{mg}$ TDS unless haemodynamic status, renal function and potential drug interactions mandated a dose reduction. Follow-up 2D echocardiography was arranged in all surviving (21) patients during inpatient stay 48-72 hours after commencement of treatment to assess right ventricular function and PASP. RVD was documented in eight and improved in all. PASP was initially elevated (range 35-80 $\mathrm{mmHg}$ ) in 19 patients and subsequently reduced in 18 (range 2-41 $\mathrm{mmHg}$; median reduction $12 \mathrm{mmHg}$ ). A dilated right ventricle was present in 14 patients and improved in 11.

\section{Pneumonitis and evolving pulmonary fibrosis}

Fifteen patients (34\%) received intravenous (IV) methylprednisolone $1 \mathrm{~g}$ daily for 3 days followed by oral prednisolone $40 \mathrm{mg}$ once a day reducing by $5 \mathrm{mg}$ after every 3 days to zero. Overall survival in this group of patients was $67 \%$ (10 out of 15). Five of these patients also had pulmonary thrombosis (four peripheral and one proximal: all patients with peripheral thrombosis survived). The first five patients who received IV methylprednisolone died. They were at the end stage in their illness and received IV methylprednisolone as a last resort. We subsequently reconsidered the indication for high-dose steroid therapy to reflect the early onset of evolving fibrosis (ground glass shadowing, peripheral airspace dilatation and traction bronchiectasis) on thoracic $\mathrm{CT}$. The next 10 patients who received IV methylprednisolone were identified early in their disease. Sepsis was ruled out before starting treatment. Repeat thoracic CTs were performed which showed improvement (Fig 2). No patient developed sepsis as a complication of high-dose steroid therapy. One patient developed an acute on chronic subdural haematoma 2 weeks after methylprednisolone therapy and completely recovered without neurosurgical intervention. Another patient had encephalopathy diagnosed 7 days after commencing methylprednisolone therapy. Following neurological review, he recovered fully without any specific intervention. All 10 patients demonstrated an excellent improvement in respiratory status clinically and radiologically.

Seven patients (five men and two women) transferred from other hospitals were receiving steroid therapy according to different protocols. We did not make any change to this treatment. Four patients (three men and one woman) were on a previously described protocol and three patients (two men and one woman) received oral prednisolone (varying between $20-40$ mg once a day in a weaning dose). ${ }^{13}$ One patient died (one man) and six patients (five men and one woman) survived. Three patients have ongoing significant lung injury.

\section{Thoracic CT changes}

The most common changes on thoracic CT were ground-glass opacities in 39 patients $(86 \%)$, consolidation in 36 patients (79\%) and pleural effusions in 18 patients (40\%). Some patients were also noted to have more unusual presentations including three with pneumatoceles. Some patients had more than one abnormality (Fig 3). 
Fig 2. Thoracic computed tomography before (a) and after (b) pulse intravenous methylprednisolone.
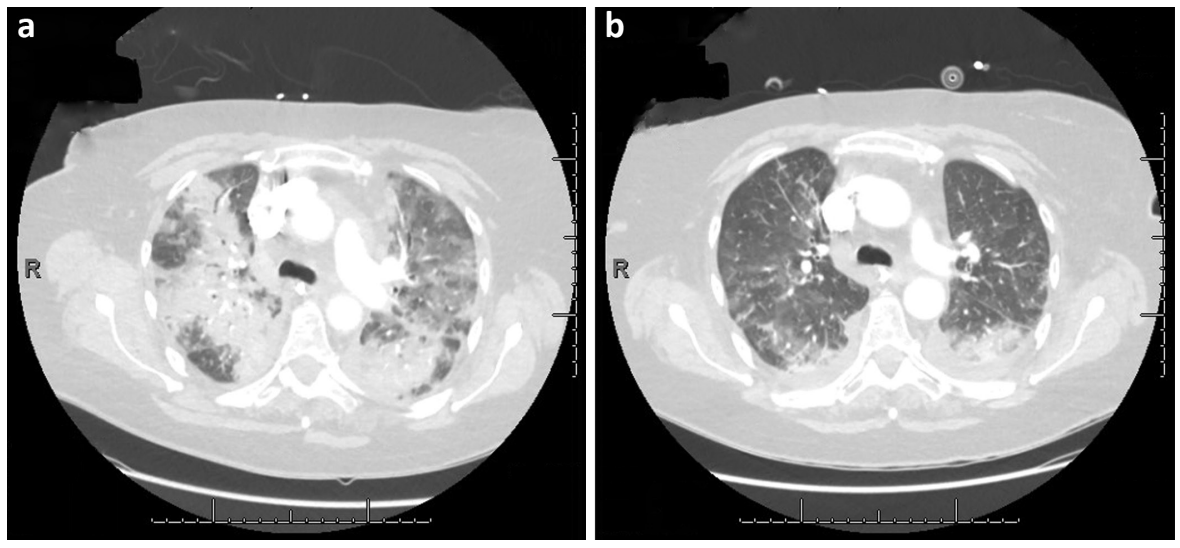

\section{Other conditions}

Airway complications were seen in five patients comprising of tracheal tear, tracheal granulation tissue, bronchomalacia, tracheal diverticulum and oedematous glottitis. These complications were noted on thoracic CT and were also confirmed by bronchoscopy. All were transferred from other hospitals. One had an iatrogenic $3 \mathrm{~cm}$ tracheal tear associated with intubation using a stylet and presented with pneumomediastinum. He was managed conservatively and follow-up rigid bronchoscopy confirmed defect closure. A second patient developed granulation tissue formation superior to the site of entry of the tracheostomy tube insertion site and this was successfully resected at rigid bronchoscopy prior to decannulation. Another patient developed left bronchomalacia and he was decannulated following successful insertion of a left main bronchial stent. The fourth patient has a persistent tracheal diverticulum that has been kept under prospective surveillance. One patient who had oedematous glottitis died from multiple organ failure.
Renal impairment was observed in eighteen patients (41\%) during the course of their disease as defined by a glomerular filtration rate of less than $60 \mathrm{~mL} / \mathrm{min} / 1.73 \mathrm{~m}^{2}$. There were 17 men and one woman. Eight patients (seven men and one woman) required renal replacement therapy. Two patients (two men) who received renal replacement therapy died. Eight of the other patients died. Overall, mortality in this subset of patients was $55 \%$.

Sepsis was suspected in 26 patients (59\%). In 10 of these patients (38\%), although sepsis was suspected, no source or pathogenic organism was identified and broad-spectrum antimicrobial agents were prescribed. Eighteen patients (41\%) of the 44 patients in our group did not exhibit signs of sepsis during their follow-up with us (Fig 4).

\section{Feedback}

There was strong positive feedback from our colleagues regarding support, education, uniformity of care across units and a unifying
Fig 3. Observed changes on thoracic computed tomography.

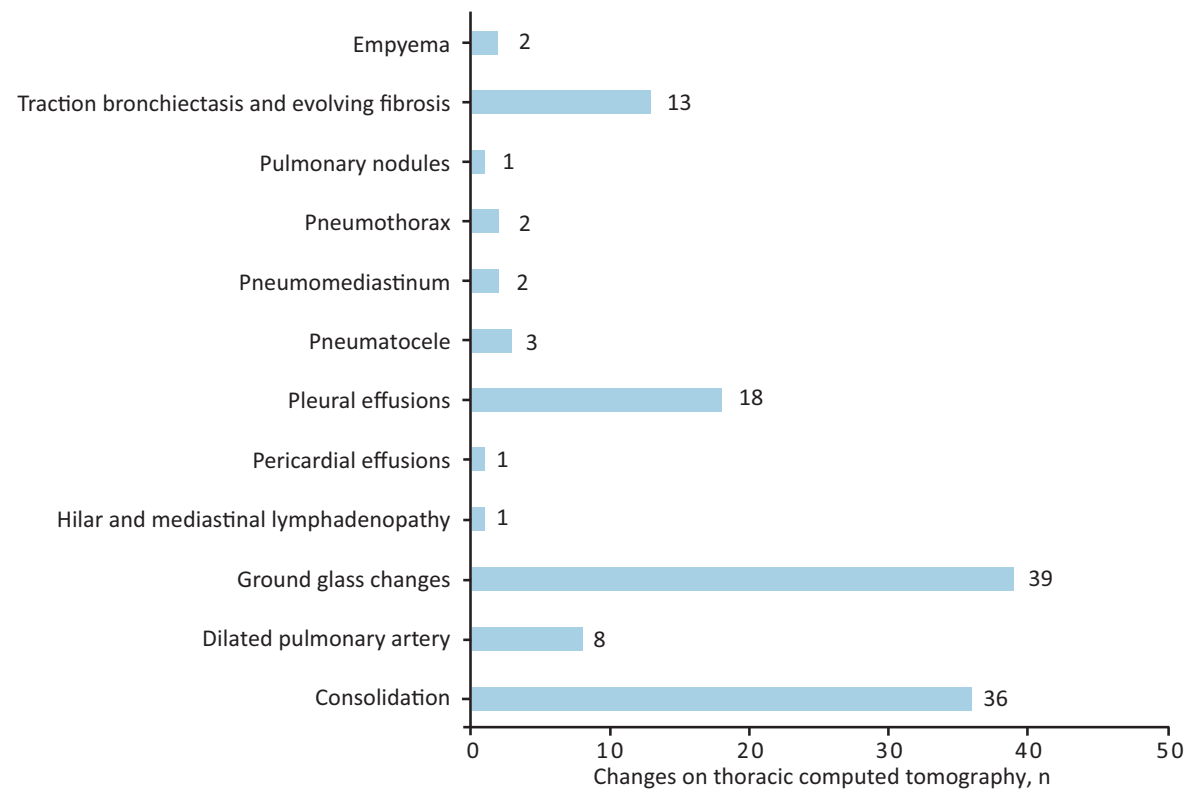




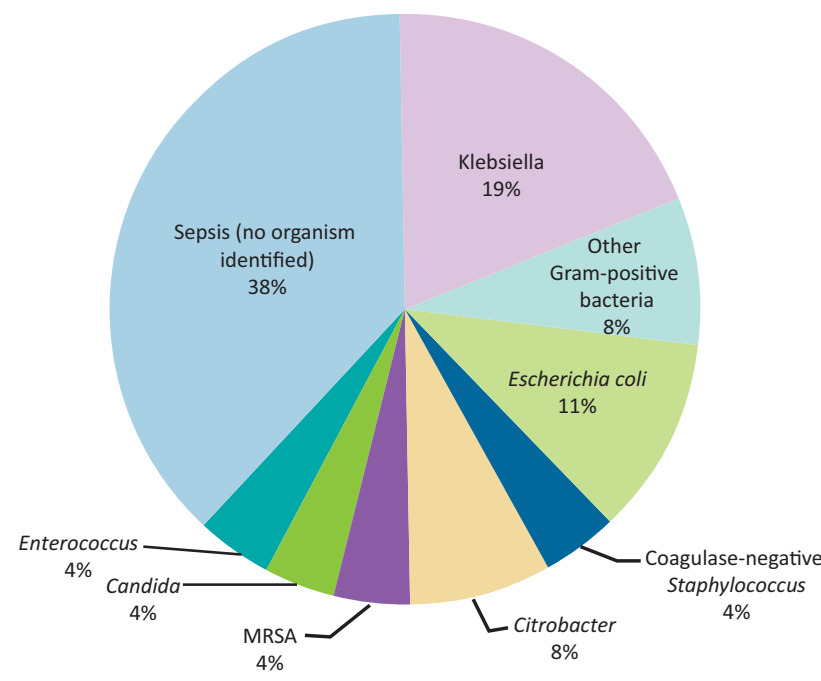

Fig 4. Representation of sepsis and causative microorganism. MRSA = Methicillin-resistant Staphylococcus aureus.

belief that despite the uncertainties and lack of knowledge of this disease that everything that could be done was being done.

\section{Discussion}

Our experience suggests that critically ill patients with SARS-CoV-2 infection benefit from a bespoke understanding of the different pathologies that may be contributing to their clinical presentation. We encountered pulmonary thrombosis in $52 \%$ of our patients, which is in line with recent reports. ${ }^{2,3}$ The majority were peripheral (perhaps in situ), but eight had proximal thrombotic elements. We did not identify a source of embolism for these patients.

We were encouraged by the use of sildenafil in our patients with pulmonary thrombosis and $\mathrm{PH}$. The concept of sildenafil use was based on experience with managing patients with pulmonary thrombosis and $\mathrm{PH}$ in the non-COVID-19 setting. ${ }^{14}$ We also believed that this agent could be particularly useful in vasodilating peripheral pulmonary vessels. An improvement in echocardiography assessment was observed in all patients who received sildenafil and we did not encounter side effects. We believe that further studies are required to define the role for sildenafil in COVID-19 patients with PH whether or not this is associated with pulmonary thrombosis.

The precise indication for steroid therapy in this condition is unclear. We considered high-dose methylprednisolone therapy followed by a tapering dose of oral prednisolone as is prescribed for patients who develop acute lung injury or who have acute rejection following lung transplantation. ${ }^{10,11}$ We report encouraging experience in 10 consecutive patients who demonstrated a significant improvement in respiratory status following this treatment. Upon reflection, the first five patients who received this treatment were at an advanced stage of their illness and had multiple comorbidities which would render the likelihood of response to be low. We would not now consider high-dose steroid therapy for this group. Two patients had a neurological presentation while receiving steroid therapy. We are unclear as to whether there is a link, but each patient made a complete recovery without specific intervention. None developed sepsis. We encountered seven patients who were on other steroid regimens started at their referring centre and these were not altered. Four patients were on a Meduri protocol and three were on reducing oral prednisolone commencing at a dose of $40 \mathrm{mg}$ daily. ${ }^{13}$ One of these patients died and three of the survivors have ongoing significant lung injury. We believe that further investigation is warranted to confirm whether high-dose IV methylprednisolone therapy ( $1 \mathrm{~g}$ daily for 3 consecutive days) followed by a reducing dose of oral prednisolone will reverse or moderate the evolution of pulmonary fibrosis associated with COVID-19 infection.

Five patients (11\%) had airway complications and it is therefore important to recognise that airway complications can occur in this cohort of patients and that specialist management may be required.

Forty-one per cent of our patients developed significant renal impairment during the course of their disease with a mortality of $55 \%$. We hope that further experience in the management of these complex patients will clarify our understanding of the nature of acute renal impairment in these patients, which may be multifactorial. ${ }^{15}$

We noted a wide array of abnormal findings on thoracic CT. Three patients had pneumatoceles which we believe reflected the effects of mechanical ventilation together with COVID-19. No specific treatment was given. Bacterial and fungal infection was excluded. The presence of pneumomediastinum and surgical emphysema was a further alert to a large airway complication. One of the patients who developed a left empyema had previously received catheter-directed thrombolysis for extensive right-sided pulmonary thrombosis. He received antibiotic therapy and CT-guided drainage and made a full recovery. We were not able to clarify on imaging as to whether the thrombotic elements observed reflected embolism or in situ thrombosis.

The mortality for the patients in our population was $34 \%$ and this represented a significant reduction in mortality $(p=0.012)$. We believe that this reflected the specialist approach to diagnose and treat the diverse pathologies encountered in these patients. Thirty-one of our patients were from BAME backgrounds and mortality was significantly higher in this group as compared with white $(p=0.035)$.

There was no difference between those who survived and those who died while on mechanical ventilatory support with regard to age and gender $(p=0.69)$. However, those who died spent significantly less time on the ventilator $(p<0.001)$. It is possible that these patients had a more aggressive disease presentation. However, it is also possible that those patients who had prolonged periods of mechanical ventilatory support had, with our improving experience, a greater appreciation of their underlying disease, had more specific diagnoses made and received appropriate targeted treatment. The improved outcome and successful weaning from mechanical ventilatory support may have reflected this.

The Intensive Care National Audit \& Research Centre (ICNARC) report described 1,188 critically ill patients with COVID-19 in south London ICUs and 10,624 patients in other ICUs throughout the UK. The median age on admission to ICU was 58 years and 60 years, respectively. There were $70.9 \%$ and $70.2 \%$ men, and $29.1 \%$ and $29.8 \%$ women in each group. ${ }^{16}$ In the south London group, $78.8 \%$ of patients had received advanced (mechanical ventilatory) respiratory support and $72.1 \%$ had received this treatment in the national group. Approximately $40 \%$ of patients overall died in each group and those who survived spent longer 


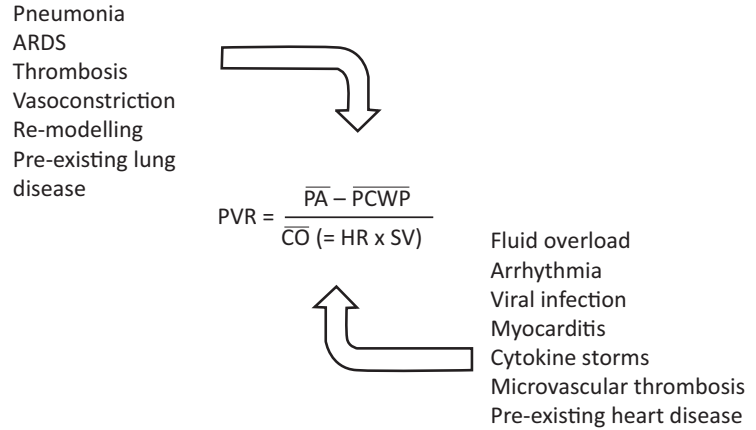

Fig 5. Factors effecting pulmonary vascular resistance. ARDS = acute respiratory distress syndrome; $\mathrm{CO}=$ cardiac output; $\mathrm{HR}=$ heart rate; $\mathrm{PA}=$ pulmonary artery pressure; PCWP = pulmonary capillary wedge pressure; $\mathrm{PVR}=$ pulmonary vascular resistance; $\mathrm{SV}=$ stroke volume.

time in ICU. Mortality for non-White was $55.3 \%$ in the south London group and $33.8 \%$ in the other group. Forty-six point nine per cent of patients in the south London group and $48 \%$ of patients nationally who received advanced respiratory support died in ICU as did $52.6 \%$ and $57.7 \%$, respectively, of those who required renal support.

Our experience has reinforced the concept that there are many contributing factors to impaired cardiorespiratory function in COVID-19 patients and that many of these may be cocontributory to the patient's clinical presentation. We also believe that many of these challenging pathologies can adversely impact on pulmonary vascular resistance and that close attention to these factors (eg anticoagulation, the use of PVT, optimal fluid balance, rhythm control, steroid therapy and other immunological modalities) together with control of intercurrent infection and appropriate use of inotropes will contribute to a further improvement in outcomes (Fig 5).

We believe that a coordinated specialised cardiorespiratory team approach contributes significantly to the successful management and outcome of patients who are severely unwell with COVID-19 and offers an important platform for continuity of patient care, education and staff wellbeing.

\section{Acknowledgements}

We are grateful to Dr D Spray and Dr R Badir for the support given to develop our team and to all our excellent medical and nursing colleagues in intensive care. Also, we are grateful to Dr O Valencia for statistical advice and to Dr S Grubnic for radiological support.

\section{References}

1 Li H, Liu L, Zhang D et al. SARS-CoV-2 and viral sepsis: observations and hypotheses. Lancet 2020;395:1517-20.

2 Bompard F, Monnier H, Saab I et al. Pulmonary embolism in patients with Covid-19 pneumonia. Eur Respir J 2020;56:2001365.

3 Carsana L, Sonzogni A, Nasr A et al. Pulmonary post-mortem findings in a series of COVID-19 cases from northern Italy: a twocentre descriptive study. Lancet Infect Dis 2020;20:1135-40.

4 Doyen D, Moceri P, Ducreux D, Dellamonica J. Myocarditis in a patient with COVID-19: a cause of raised troponin and ECG changes. Lancet. 2020;395:1516.

5 Tersalvi G, Vicenzi M, Calabretta D et al. Elevated troponin in patients with coronavirus disease 2019: possible mechanisms. J Card Fail 2020;26:470-5.

6 Horby P, Lim WS, Emberson JR et al. Dexamethasone in hospitalized patients with COVID-19 - preliminary report. N Engl J Med 2020:NEJMoa2021436 [Epub ahead of print].

7 Madden BP. Treatment of pulmonary hypertension. Springer, 2015.

8 Bastiaenen R, Madden BP. Should all patients with myocarditis have an endomyocardial biopsy? Cardio Debate, 2017. www.cardiodebate.com/2017/05/08/should-all-patients-with-myocarditis-havean-endomyocardial-biopsy [Accessed 15 July 2020].

9 Madden BP. Evolutional trends in the management of tracheal and bronchial injuries. J Thorac Dis 2017;9:E67-70.

10 Madden BP, Hodson ME, Tsang V et al. Intermediate-term results of heart-lung transplantation for cystic fibrosis. Lancet 1992;339:1583-7.

$11 \mathrm{Vij}$ R, Strek ME. Diagnosis and treatment of connective tissue disease-associated interstitial lung disease. Chest 2013;143:814-24.

12 Bradley B, Branley HM, Egan J] et al. Interstitial lung disease guideline: the British Thoracic Society in collaboration with the Thoracic Society of Australia and New Zealand and the Irish Thoracic Society. Thorax 2008;63 (Suppl 5):v1-58.

13 Meduri GU, Siemieniuk RAC, Ness RA et al. Prolonged low-dose methylprednisolone treatment is highly effective in reducing duration of mechanical ventilation and mortality in patients with ARDS. J Intensive Care 2018;6:53.

14 Galiè N, Humbert M, Vachiery JL et al. ESC/ERS Guidelines for the diagnosis and treatment of pulmonary hypertension. Eur Heart J 2016:37:67-119.

15 Ronco C, Reis T, Husain-Syed F. Management of acute kidney injury in patients with COVID-19. Lancet Respir Med 2020;8:738-42.

16 Intensive Care National Audit \& Research Centre. ICNARC report on COVID-19 in critical care: London - South Operational Delivery Network. ICNARC, 2020.

Address for correspondence: Prof Brendan Madden, Cardiothoracic Medicine, St George's Hospital, Blackshaw Road, Tooting, London SW17 0QT, UK. Email: brendan.madden@stgeorges.nhs.uk 